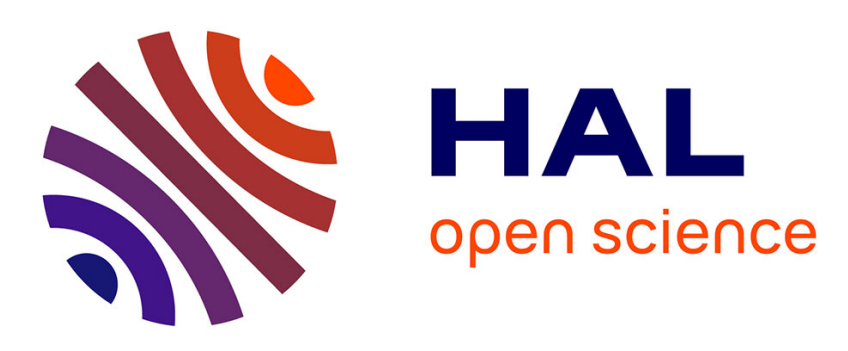

\title{
Influence of Gas Turbulence on the Instability of an Air-Water Mixing Layer
}

Jean-Philippe Matas, Sylvain Marty, Mohamed Seydou Dem, Alain H. Cartellier

\section{- To cite this version:}

Jean-Philippe Matas, Sylvain Marty, Mohamed Seydou Dem, Alain H. Cartellier. Influence of Gas Turbulence on the Instability of an Air-Water Mixing Layer. Physical Review Letters, 2015, 115 (7), 10.1103/PhysRevLett.115.074501 . hal-01212562

\section{HAL Id: hal-01212562 https://hal.science/hal-01212562}

Submitted on 6 Oct 2015

HAL is a multi-disciplinary open access archive for the deposit and dissemination of scientific research documents, whether they are published or not. The documents may come from teaching and research institutions in France or abroad, or from public or private research centers.
L'archive ouverte pluridisciplinaire HAL, est destinée au dépôt et à la diffusion de documents scientifiques de niveau recherche, publiés ou non, émanant des établissements d'enseignement et de recherche français ou étrangers, des laboratoires publics ou privés. 


\title{
Influence of gas turbulence on the instability of an air-water mixing layer
}

\author{
Jean-Philippe Matas, Sylvain Marty, Mohamed Seydou Dem \& Alain Cartellier \\ Université Grenoble Alpes, LEGI, F-38000 Grenoble, France \\ CNRS, LEGI, F-38000 Grenoble, France
}

(Dated: June 9, 2015)

\begin{abstract}
We present the first evidence of the direct influence of gas turbulence on the shear instability of a planar air-water mixing layer. We show with two different experiments that increasing the level of velocity fluctuations in the gas phase continuously increases the frequency of the instability, up to a doubling of frequency for the largest turbulence intensity investigated. A modified spatiotemporal stability analysis taking turbulence into account via a simple Reynolds stress closure provides the right trend and magnitude for this effect.
\end{abstract}

PACS numbers: $47.20 . \mathrm{Ft}, 47.55 . \mathrm{N}-$

The fragmentation of a bulk of liquid into a spray of droplets is the goal of many applications, in particular in relation to combustion [1]. In some of these applications (e.g. turbojets, cryogenic rocket engines) liquid breakup is obtained via gas-assisted atomization: a fast gas stream destabilizes a parallel slower liquid stream. In this configuration, destabilization of the liquid is initiated by a shear instability leading to the formation of twodimensional waves $[2,3]$, see figure 1 .

The variations of the frequency of these waves with mean gas and liquid velocities $U_{G}$ and $U_{L}$ can be captured by a simple inviscid stability analysis. The most unstable frequency is then predicted to behave as $f \sim$ $\left(\rho_{G} / \rho_{L}\right) U_{G} / \delta_{G}$ where $\rho_{G}$ and $\rho_{L}$ are the gas and liquid densities and $\delta_{G}$ the vorticity thickness for the gas stream $[2,4,5]$. More sophisticated viscous approaches have recently clarified the limitations of inviscid analyses, and shown that a convective-absolute transition takes place for the typical conditions of laboratory air-water mixing layer experiments $[6,7]$. More precisely, the absolute instability can be triggered either by surface tension at larger liquid velocities or by confinement at lower liquid velocities, see Matas [8].

These works have mostly focused on explaining how the complexity of the resulting multiphase flow could be understood, and ultimately modelled via a succession of instabilities. The goal is typically to predict droplet size or velocity distributions in terms of the mean gas and liquid velocities, vorticity thickness, and geometry of the injector $[4,9,10]$. In the present letter, we present new experimental data proving that mean quantities are not sufficient to determine the features of the shear instability, and hence of the resulting spray: we demonstrate that velocity fluctuations in the gas stream play a key role in frequency selection.

The experimental set-up is shown on figure 2: the water and air streams are injected in rectangular channels (10 $\mathrm{cm}$ width, $5 \mathrm{~cm}$ height for the liquid and $9.5 \mathrm{~cm}$ height for the gas) through honeycombs and convergents of same width and final heights $H_{G}=1 \mathrm{~cm}$ for the gas and $H_{L}=1 \mathrm{~cm}$ for the liquid. Water comes from an overflowing reservoir located above the experiment, air is provided via a blower and heat exchanger. The level

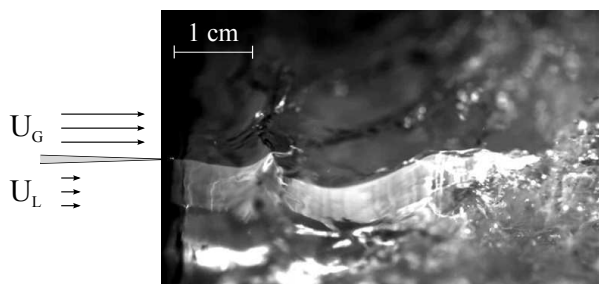

FIG. 1. Destabilization of a slow liquid layer by a fast gas stream (Argon laser vertical slice + white light), $U_{G}=23 \mathrm{~m} / \mathrm{s}$ and $U_{L}=0.19 \mathrm{~m} / \mathrm{s}$ : large wavelength waves form and are subsequently atomized into droplets.

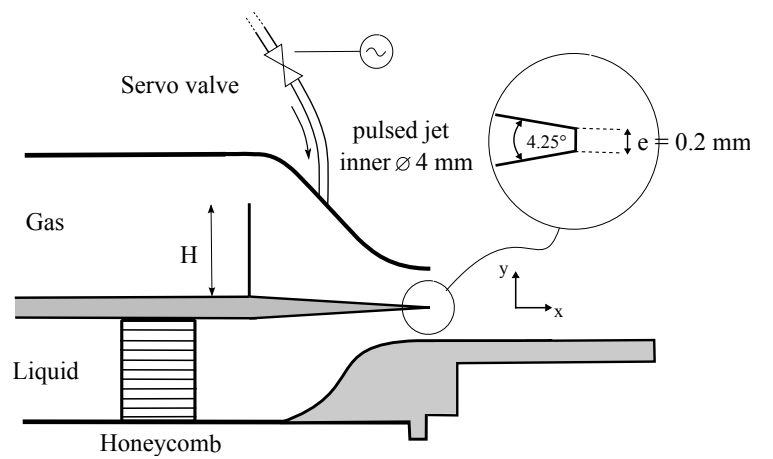

FIG. 2. Experimental set-up used in the present study. Two forcing methods can be used: either an obstruction of height $H$ in the gas channel, or injection of a pulsed jet through the gas convergent.

of fluctuation in the gas phase can be forced with two alternative methods. The first method consists in inserting just upstream of the gas convergent an obstruction of varying height $H$ : the air flow can only pass above this wall, see figure 2. The second method consists in sending through the outer wall of the gas convergent a pulsed jet normal to the axis of the injector. The pulsed jet is controlled with a servo valve (Asco Sentronic 601): by feeding the valve with a sine wave signal of varying voltage and frequency, we can modulate the flow rate and frequency of this gas jet.

Vertical hot-wire velocity profiles are carried out for 

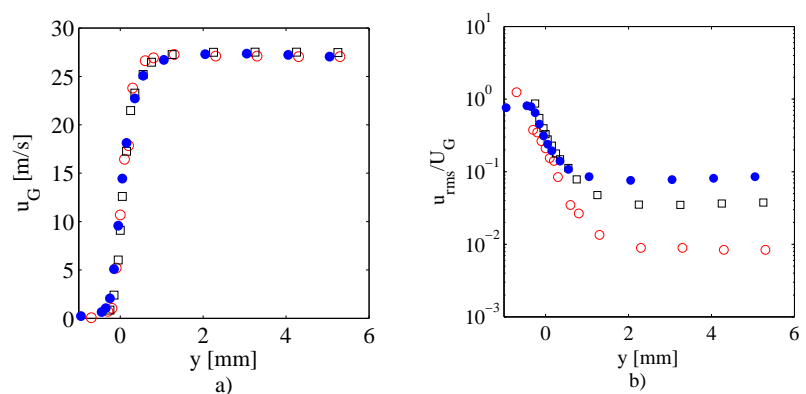

FIG. 3. (color online) Hot-wire velocity profiles for $U_{G}=$ $27 \mathrm{~m} / \mathrm{s}$ and varying obstruction heights $H: \circ: H=0 ; \square$ : $H=5.6 \mathrm{~cm} ; \bullet: H=8 \mathrm{~cm}$; a) Mean velocity profile. b) Turbulence intensity $u_{r m s} / U_{G}$.

each of the forcing conditions in the gas stream. Measurements are carried out in a vertical plane located at less than $500 \mu \mathrm{m}$ downstream of the splitter plate. In order to ensure the flow around the hot-wire probe is monophasic, the lower liquid channel is dried for this measurement. Figure 3a shows the comparison of three mean velocity profiles obtained without obstruction and with two different obstructing heights $H$. Figure 3b compares turbulence intensity $u_{r m s} / U_{G}$ profiles for the same three cases. Position $\mathrm{y}=0$ indicates the position of the splitter plate. Data show that while mean velocity profiles are virtually undistinguishable, and hence associated $\delta_{G}=600 \pm 20 \mu \mathrm{m}$ identical, the turbulence intensity in the middle of the gas channel varies from $0.8 \%$ in the unobstructed case to $3.5 \%$ for $H=5.6 \mathrm{~cm}$ and to $8 \%$ for $H=8 \mathrm{~cm}$.

The frequency of the surface instability is measured with a phase detection optical probe [11]: the probe is positioned $2 \mathrm{~cm}$ downstream of the splitter plate, with its tip at the height of the splitter plate. A laser signal is sent into the probe: the variations of the reflected signal (sampled at $1 \mathrm{kHz}$ ) directly detect the interception of liquid waves at the tip. A spectrum of this signal is then computed and averaged with Matlab (pwelch function), with a resolution of $0.25 \mathrm{~Hz}$. Figure 4a compares the resulting spectra for $H=0$ (solid line) and $H=8 \mathrm{~cm}$ (dotted line), for fixed $U_{G}$ and $U_{L}$ : a peak and its harmonics are clearly visible in both spectra. The peak frequency is $f=25.6 \mathrm{~Hz}$ for $H=0$, and $f=42.1 \mathrm{~Hz}$ for $H=8 \mathrm{~cm}$ : frequency is larger when the gas channel is obstructed.

We then carry out frequency measurements for several values of $H$ between $H=0$ and $H=8.6 \mathrm{~cm}$, for four sets of fixed $U_{G}$ and $U_{L}$. Mean gas velocity is adjusted before each measurement with a Pitot tube, to ensure $U_{G}$ remains within $\Delta U_{G}<0.5 \mathrm{~m} / \mathrm{s}$ from its expected value. Figure 5 a shows that frequency increases steadily when $H$ is increased, for all gas and liquid velocities investigated. The error bars correspond to the width of the peak in the spectrum. Figure $5 \mathrm{~b}$ shows the ratio of these frequencies at finite $H$ to frequency when $H=0$, noted $f_{0}$, as a function of the mid-channel turbulence in-
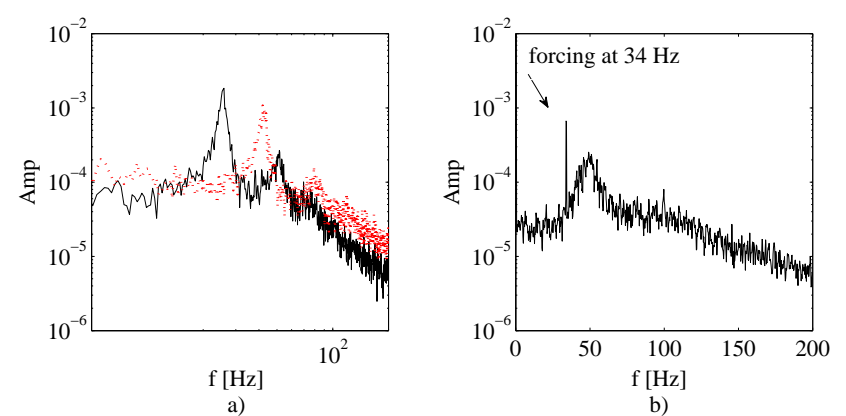

FIG. 4. Spectrum of optical probe signal for $U_{G}=27 \mathrm{~m} / \mathrm{s}$ and $U_{L}=0.28 \mathrm{~m} / \mathrm{s}$. a) Solid line: spectrum for $H=0$; dotted line: spectrum for $H=8 \mathrm{~cm}$. The frequency peak is shifted to larger values. b) $H=0$ and forcing with a pulsed jet of frequency $f=34 \mathrm{~Hz}$ and $u_{r m s} / U_{G}=0.068$. The frequency of the instability, $f \approx 49 \mathrm{~Hz}$, is distinct from the pulsed jet frequency.
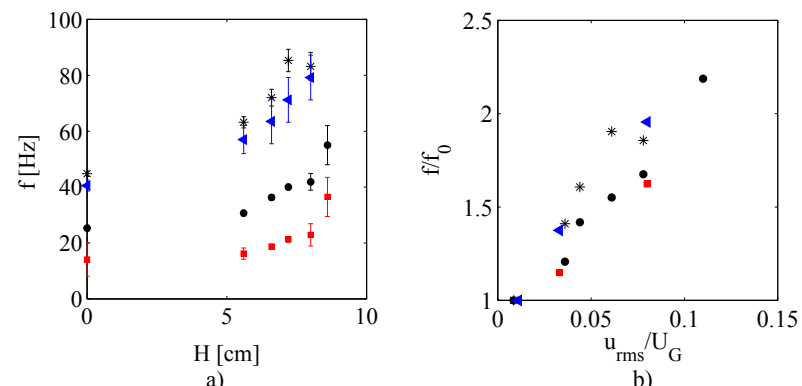

FIG. 5. (color online) a) Frequency of shear instability as a function of height of obstruction $H$ for $\bullet U_{G}=27 \mathrm{~m} / \mathrm{s}$ and $U_{L}=0.28 \mathrm{~m} / \mathrm{s} ; * U_{G}=27 \mathrm{~m} / \mathrm{s}$ and $U_{L}=0.95 \mathrm{~m} / \mathrm{s} ;$ $U_{G}=17.5 \mathrm{~m} / \mathrm{s}$ and $U_{L}=0.28 \mathrm{~m} / \mathrm{s} ; \triangleleft U_{G}=40 \mathrm{~m} / \mathrm{s}$ and $U_{L}=0.28 \mathrm{~m} / \mathrm{s}$. b) Ratio of frequency of shear instability to frequency for $H=0$, as a function of mid-channel turbulence intensity: the data are collapsed.

tensity: all series are collapsed. For all data investigated here frequency is doubled when turbulence intensity is of the order of $10 \%$.

In order to show that the observed impact on frequency is not specific to the previous forcing method, we now study the impact of a totally different forcing: we keep $H=0$, but as described on figure 2 inject a pulsed air jet through the outer wall of the gas convergent. Three frequencies are used for the pulsing: $f=17 \mathrm{~Hz}, f=34 \mathrm{~Hz}$, and $f=70 \mathrm{~Hz}$. As with the first method, hotwire measurements are carried out to check that mean profiles and hence $\delta_{G}$ values are not modified by the forcing. Optical probe spectra obtained with the pulsed jet method are quite similar to spectra obtained with the obstruction wall method, except for an additional sharp frequency peak at the pulsed jet frequency $(17 \mathrm{~Hz}, 34 \mathrm{~Hz}$ or $70 \mathrm{~Hz})$, see figure $4 \mathrm{~b}$. Figure $6 \mathrm{a}$ shows the variations of wave frequency as a function of the turbulence intensity at midheight of the gas channel. We find the forcing has 


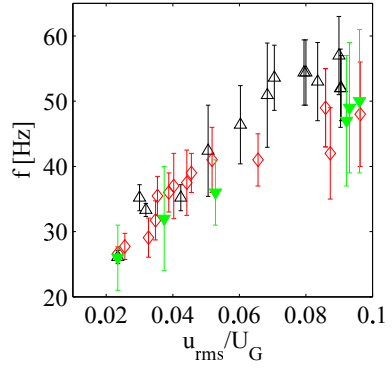

a)



b)
FIG. 6. a) Frequency of shear instability as a function of midheight gas channel turbulence intensity, for fixed $U_{G}=$ $27 \mathrm{~m} / \mathrm{s}$ and $U_{L}=0.28 \mathrm{~m} / \mathrm{s}$ and for forcing with a pulsed jet. Forcing jet frequency is: $\nabla: 17 \mathrm{~Hz} ; \triangle: 34 \mathrm{~Hz} ; \diamond 70 \mathrm{~Hz}$. b) Ratio of frequency of shear instability to frequency without forcing $f_{0}$, as a function of mid-channel turbulence intensity: the pulsed jet data are superposed to the data of figure 5 (same symbols).

an impact similar to the one observed in figure 5b: the instability frequency increases continuously with increasing turbulence intensity. Though the exact variations of $f$ with $u_{r m s} / U_{G}$ seem independent of the forcing jet frequency for $u_{r m s} / U_{G}<0.05$, they are more scattered for forcing intensities corresponding to $u_{r m s} / U_{G}>0.05$. In particular, for a given large turbulence intensity, the forcings at $17 \mathrm{~Hz}$ and $70 \mathrm{~Hz}$ seem to induce a lesser increase in frequency than the forcing at $34 \mathrm{~Hz}$ : This could be caused by a larger receptivity to structures generated by the $34 \mathrm{~Hz}$ jet. At any rate, one would probably have to look more closely at how the pulsed jet merges with the parallel flow for these frequencies to clarify this issue. On figure $6 \mathrm{~b}$ the data of figure $6 \mathrm{a}$ are plotted along with the data of figure $5 \mathrm{~b}$ : though obtained with totally different forcing methods, these data are relatively well collapsed, in particular for $u_{r m s} / U_{G}<0.05$.

The above data describe the impact of forced turbulence on wave frequency. We now illustrate the impact of forcing on the wavelength $\lambda$ : the waves were filmed with a high speed camera (Phantom v10) at 8600 images/s, for fixed $U_{G}=27 \mathrm{~m} / \mathrm{s}$ and $U_{L}=0.28 \mathrm{~m} / \mathrm{s}$. The forcing with the pulsed jet at $34 \mathrm{~Hz}$ is applied for four different intensities [12]. Figure 7 shows the impact on wave development when the forcing is turned on (the optical probe can be seen, located so as to intercept wavecrests): wavelength is strongly reduced when forcing is present. Measurements of $\lambda$ in table I show that $\lambda$ consistently decreases when $u_{r m s} / U_{G}$ is increased. Fast imaging allows measurement of wave velocity $u_{w}$, by following waves over a distance of $1 \mathrm{~cm}$ just after wave formation close to the splitter plate. Results of table I show that $u_{w}$ is relatively constant for all forcing conditions. The ratio $u_{w} / \lambda$ therefore increases with $u_{r m s} / U_{G}$. Frequency values derived from this ratio are in good agreement with those
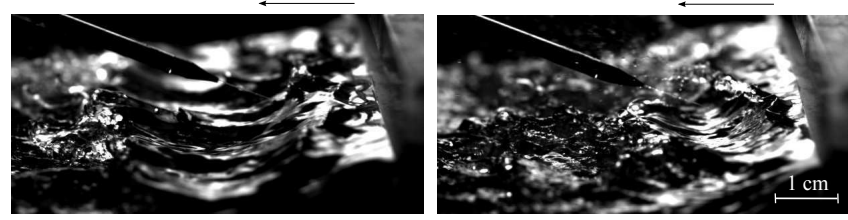

FIG. 7. Impact of forcing on the wavelength of the shear instability for $U_{G}=27 \mathrm{~m} / \mathrm{s}$ and $U_{L}=0.28 \mathrm{~m} / \mathrm{s}$; Left: no forcing; Right: forcing with pulsed jet at $34 \mathrm{~Hz}$, midchannel $u_{r m s} / U_{G}=0.09$. The forcing leads to a decrease in the wavelength.

\begin{tabular}{ccccc}
$u_{r m s} / U_{G}$ & $\lambda[\mathrm{cm}]$ & $u_{w}[\mathrm{~m} / \mathrm{s}]$ & $u_{w} / \lambda[\mathrm{Hz}]$ & $f_{\text {opt probe }}[\mathrm{Hz}]$ \\
\hline 0.023 & 3.4 & 0.84 & 25 & 25 \\
0.042 & 2.5 & 0.79 & 31.6 & 32 \\
0.068 & 2.5 & 0.9 & 36 & 37 \\
0.09 & 1.6 & 0.79 & 49.3 & 53
\end{tabular}

TABLE I. Wavelength, velocity and frequency for $U_{G}=$ $27 \mathrm{~m} / \mathrm{s}$ and $U_{L}=0.28 \mathrm{~m} / \mathrm{s}$ and for four different intensities of forcing with a pulsed jet at $34 \mathrm{~Hz}$. Measurements via high speed imaging, the uncertainty is estimated as $\Delta \lambda=$ $0.4 \mathrm{~cm}$ and $\Delta u_{w}=0.1 \mathrm{~m} / \mathrm{s}$ : when velocity fluctuations increase wavelength decreases but wave velocity remains approximately constant.

measured with the optical probe.

In order to better understand the nature of the shear instability, we now look at what stability analysis predicts. A spatiotemporal stability analysis analogous to the one used by Matas [8] is carried out: viscosity and confinement (finite $H_{L}=H_{G}=1 \mathrm{~cm}$ ) are taken into account. Vorticity thicknesses are taken equal to experimental values, with the liquid one estimated at $\delta_{L}=500 \mu \mathrm{m}$ for $U_{L}=0.28 \mathrm{~m} / \mathrm{s}$ from PIV measurements. The velocity profile is taken as a sum of error functions. Interfacial velocity is chosen so as to verify the continuity of tangential stresses, and no velocity deficit is taken into account (same expressions as in ref. [6]). For $U_{L}=0.28 \mathrm{~m} / \mathrm{s}$ and the three gas velocities investigated in figure 5 , namely $U_{G}=17.5 \mathrm{~m} / \mathrm{s}$, $U_{G}=27 \mathrm{~m} / \mathrm{s}$ and $U_{G}=40 \mathrm{~m} / \mathrm{s}$, we find that the instability is absolute, with the mechanism discussed in [8]: the pinch point arises because of the collision of the shear instability branch with a confinement branch. For these three gas velocities, the frequency at pinching is respectively 18.6, 26.2 and $37.9 \mathrm{~Hz}$ : these frequencies are close to the frequencies observed without forcing, all at $u_{r m s} / U_{G} \approx 0.01$ (see figure 5 ). The corresponding absolute growthrate $\omega_{i 0}$ is respectively 15,62 and $130 \mathrm{~s}^{-1}$. The fact that the system responds with a frequency different from that of the forcing (see spectrum of figure $4 \mathrm{~b}$ ) is consistent with the instability having an absolute nature: the system behaves as a non linear oscillator, and not as a noise amplifier. The frequency predicted by spatiotemporal stability analysis with a laminar base flow 
logically corresponds to the frequency of the oscillator at low $u_{r m s} / U_{G}$. The velocity $u_{w}$ of the associated non linear waves is expected to reach Dimotakis speed [13, 14], given by $U_{c} \approx \sqrt{\rho_{G} / \rho_{L}} U_{G}+U_{L}$, the speed of the frame in which dynamic pressures in the gas/liquid are balanced. For mean velocities $U_{G}=27 \mathrm{~m} / \mathrm{s}$ and $U_{L}=0.28 \mathrm{~m} / \mathrm{s}$ this expression gives $U_{c}=1.21 \mathrm{~m} / \mathrm{s}$, slightly larger but not far from the velocity measured in table I close to injection. The decrease in wavelength follows from the variations of $f$ and $u_{w}$.

In order to capture the impact of turbulence on the frequency of the oscillator, we follow the simple proposal made by Reynolds and Hussain [15] and assume that the additional dissipation caused by turbulence can be modelled by a constant Newtonian eddy viscosity $\nu_{t}$. We therefore look at the effect of turbulence on the frequency at the pinch point by increasing gas viscosity, namely from its molecular value for air at $\mathrm{T}=20^{\circ} \mathrm{C}$ $\nu_{G 0}=1.3610^{-5} \mathrm{~m}^{2} \mathrm{~s}^{-1}$ up to $\nu_{G}=5 \cdot 10^{-4} \mathrm{~m}^{2} \mathrm{~s}^{-1}$. In order to attempt a comparison with experimental results, we relate the turbulence intensity to the apparent gas viscosity $\nu_{G}=\nu_{0}+\nu_{t}$ by writing that $u_{r m s}^{2}=\nu_{t} U_{G} / \delta_{G}$, which gives $u_{r m s} / U_{G}=\sqrt{\left(\nu_{G}-\nu_{G 0}\right) /\left(\delta_{G} U_{G}\right)}$. Figure 8 shows that the resulting frequency prediction is in relatively good agreement with experimental data: the Newtonian eddy viscosity model, though clearly simplistic, seems to capture the overall impact of turbulence on the instability. One can note that the present stability analysis prediction (symbol $\star$ ) tends to underestimate the experimental points: this is expected since the value of $u_{r m s}$ injected in the eddy viscosity, the mid-channel value, is the smallest value in the profile of figure $3 \mathrm{~b}$. The choice of any other $u_{r m s}$ of reference in this profile, though difficult from an experimental perspective, would lead to a larger turbulent intensity, and hence to a larger predicted frequency in better agreement with experimental data. At any rate a more realistic model would have to include an eddy viscosity profile [16]. Note finally that the increase in gas viscosity has an impact on the absolute growthrate $\omega_{i 0}$, which decreases from $62 s^{-1}$ down to $35 \mathrm{~s}^{-1}$ when $\nu_{G}$ is increased up to $5.10^{-4} \mathrm{~m}^{2} \mathrm{~s}^{-1}$ : this implies that the convective/absolute transition itself will be affected for large $u_{r m s} / U_{G}$, with an increase in turbulence favouring the convective regime.

The impact of gas turbulence on this instability may be the reason of observed discrepancies between various experiments on this configuration, which all observed $f \propto U_{G} / \delta_{G}$ but with different prefactors (see e.g. figure 1 of Fuster et al. [7]): turbulence intensity in the gas stream, which was not precisely monitored, is probably the hidden parameter undermining experimental reproducibility for past two-phase mixing layer experiments.

These results provide the first evidence of a strong and controlled impact of turbulence intensity on a shear instability. We have demonstrated the robustness of this effect via two independent forcing techniques: each show up to a doubling in frequency when turbulent intensity in the incoming gas stream increases from $2 \%$ to $10 \%$. The

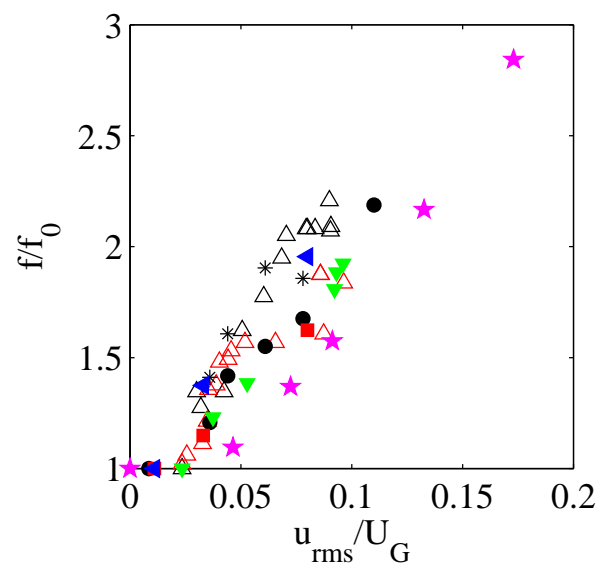

FIG. 8. (color online) Comparison of experimental data of figure $6 \mathrm{~b}$ with the prediction of stability analysis (symbol $\star$ ) for the $U_{G}=27 \mathrm{~m} / \mathrm{s}$ and $U_{L}=0.28 \mathrm{~m} / \mathrm{s}$ case.

break-up of the instability waves has been recognized as central in drop formation $[4,9,17]$ : the present results therefore reassert the relevance of internal flow characteristics on assisted atomization, beyond the already established role of $\delta_{G}$.

It will next be crucial for improving applications to assess how upstream turbulence, via its effect on the shear instability, impacts drop sizes. More precisely, the latter are already known to depend on non-linear interface deformation and gas turbulence generation in the two-phase mixing layer [18-20]: the open question is how these effects combine in the atomization process, and what their respective influence is.

The laboratory LEGI is part of the LabEx Tec 21 (Investissements d'Avenir - grant agreement $\mathrm{n}^{\circ} \mathrm{ANR}-11-$ LABX-0030).
[1] A. Lefebvre, Atomization and Sprays (New York: Hemisphere, 1989).

[2] L. Raynal, E. Villermaux, J. C. Lasheras, and E. J. Hopfinger, in Proceedings of the 11th Symp. on Turbulent Shear Flows, Vol. 3 (INP-CNRS-UJF, Grenoble, France, 1997) pp. 27.1-27.5.

[3] J. C. Lasheras and E. J. Hopfinger, Annu. Rev. Fluid Mech. 32, 275 (2000).
[4] P. Marmottant and E. Villermaux, J. Fluid Mech. 498, 73 (2004).

[5] J.-P. Matas, S. Marty, and A. Cartellier, Phys. Fluids 23, 094112 (2011).

[6] T. Otto, M. Rossi, and T. Boeck, Phys. Fluids 25, 032103 (2013).

[7] D. Fuster, J.-P. Matas, S. Marty, S. Popinet, J. Hoepffner, A. Cartellier, and S. Zaleski, J. Fluid Mech. 
736, 150 (2013).

[8] J.-P. Matas, J. Fluid Mech. 768, 375 (2015).

[9] M. Hong, A. Cartellier, and E. J. Hopfinger, in 4th International Conference on Launcher Technology Space Launcher Liquid Propulsion. Liège, Belgium (Belgium CNES Publ., 2002).

[10] C. Varga, J. C. Lasheras, and E. J. Hopfinger, J. Fluid Mech. 497, 405 (2003).

[11] M. Hong, A. Cartellier, and E. J. Hopgfinger, Int. J. Multiphase Flow 30, 615 (2004).

[12] See Supplemental Material at [URL will be inserted by publisher], where these high-speed videos have been deposited.
[13] P. E. Dimotakis, AIAA J 24, 1791 (1986).

[14] J. Hoepffner, R. Blumenthal, and S. Zaleski, Physical Review Letters 106, 104502 (2011).

[15] W. Reynolds and A. Hussain, J. Fluid Mech. 54, 263 (1972).

[16] L. Ó Náraigh, P. D. M. Spelt, and S. J. Shaw, J. Fluid Mech. 714, 58 (2013).

[17] J. John Soundar Jerome, S. Marty, J.-P. Matas, S. Zaleski, and J. Hoepffner, Phys. Fluids 25, 112109 (2013).

[18] U. Shavit, Experiments in Fluids 31 (2001).

[19] A. Kourmatzis and A. R. Masri, Experiments in Fluids 55:1659 (2014)

[20] A. Kourmatzis and A. R. Masri, J. Fluid Mech. 764, 95 (2015). 